\title{
antioxidants
}

ISSN 2076-3921

www.mdpi.com/journal/antioxidants

Article

\section{Changes of Major Antioxidant Compounds and Radical Scavenging Activity of Palm Oil and Rice Bran Oil during Deep-Frying}

\author{
Azizah Abdul Hamid ${ }^{1, *}$, Mohd Sabri Pak Dek ${ }^{1}$, Chin Ping Tan ${ }^{2}$, Mohd Asraf Mohd Zainudin ${ }^{1}$ \\ and Evelyn Koh Wee Fang ${ }^{1}$
}

1 Department of Food Science, Faculty of Food Science and Technology, Universiti Putra Malaysia, UPM Serdang, Selangor 43400, Malaysia; E-Mails: mpakdek@uoguelph.ca (M.S.P.D.); asraf_zainudin@yahoo.com (M.A.M.Z); evelyn_kwf@hotmail.com (E.K.W.F.)

2 Department of Food Technology, Faculty of Food Science and Technology, Universiti Putra Malaysia, UPM Serdang, Selangor 43400, Malaysia;

E-Mail: tancp@upm.edu.my

* Author to whom correspondence should be addressed; E-Mail: azizahah@upm.edu.my;

Tel.: +603-8946-8374; Fax: +603-8945-3552.

Received: 18 March 2014; in revised form: 29 April 2014 / Accepted: 15 May 2014 /

Published: 10 July 2014

\begin{abstract}
Changes in antioxidant properties and degradation of bioactives in palm oil (PO) and rice bran oil (RBO) during deep-frying were investigated. The alpha $(\alpha)$-tocopherol, gamma $(\gamma)$-tocotrienol and $\gamma$-oryzanol contents of the deep-fried oils were monitored using high performance liquid chromatography, and antioxidant activity was determined using 2-diphenyl-1-picryl hydrazyl (DPPH) radical scavenging activity. Results revealed that the antioxidant activity of PO decreased significantly $(p<0.05)$, while that of RBO was preserved after deep-frying of fries. As expected, the concentration of $\alpha$-tocopherol in PO and $\gamma$-tocotrienol in both PO and RBO decreased significantly $(p<0.05)$ with increased frying. Results also showed that $\gamma$-tocotrienol was found to be more susceptible to degradation compared to that of $\alpha$-tocopherol in both PO and RBO. Interestingly, no significant degradation of $\alpha$-tocopherol was observed in RBO. It is suggested that the presence of $\gamma$-oryzanol and $\gamma$-tocotrienol in RBO may have a protective effect on $\alpha$-tocopherol during deep-frying.
\end{abstract}


Keywords: vegetable oils; deep-frying; kinetic degradation; free radical scavenging; oil antioxidants

\section{Introduction}

Vegetable oils are generally known to be healthy, due to being high in unsaturated fatty acid and other various phytochemical compounds. Palm oil (PO) is produced from the fruit bunches of the tree oil palm (Elaeis guineensis) and has been used for food preparations, as well as other commercial applications in many parts of the world. Yellow palm olein is well known for its high concentration of lipophilic antioxidants, in particular, tocopherols and tocotrienols, as compared to that of other vegetable oils, but lacking in carotenoids, which are removed during the refining process [1]. In addition, PO contains an equal ratio of unsaturated to saturated fatty acids, making it a versatile oil for commercial usage without involving major modification processes [2].

Rice bran oil (RBO) has been used as cooking oil in some parts of the world, particularly in Japan and India [3]. Rice bran oil has been reported to be an excellent source of antioxidants, such as tocopherols, tocotrienols and oryzanols [4]. Generally, RBO consists of up to $1.5 \%$ of oryzanol depending on the variety of the rice and is well known for its stability as a frying oil $[5,6]$. The role played by both tocols and oryzanol in improving lipid stability and human health is fascinating, although the use of rice bran oil is not as popular as that of other vegetables oils [7,8]. Studies have shown that tocopherols, tocotrienol, carotenoids and oryzanols exhibited potent antioxidant properties in both in vivo and in vitro systems $[9,10]$.

Deep-frying is a convenient and rapid process to produce fried foods with intense flavor [11]. In this process, food is immersed in edible oil at a maintained temperature $\left(150\right.$ to $\left.200{ }^{\circ} \mathrm{C}\right)$ for a specific period of time [12]. However, in certain conditions, instead of the desirable intensive flavor, undesirable off-odor and toxic compounds from lipid peroxidation can also be produced during deep-frying, which is normally associated with the type of oil used. The alteration of flavor and oil quality during deep-frying can occur via the hydrolysis, oxidation and polymerization of the oil used as a result of the long exposure to high temperature [13]. Several in vivo studies revealed the existence of the relationship between deep-frying oil quality intake with oxidative stress level. The intake of such an altered oil quality could affect both the plasma and mitochondrial membrane [14,15].

Although oils, like $\mathrm{PO}$ and $\mathrm{RBO}$, contain bioactive compounds that can protect lipids from oxidation, the reaction can still occur during deep-frying, which results from the degradation of the bioactive compounds, as affected by exposure to high temperature $[3,13]$. Therefore, it is important to evaluate the stability and degradation pattern of bioactive compounds in the oils during food preparations, in an effort to get a better picture on effective oil usage before complete depletion of bioactive compounds occur. The consumer can then gain the beneficial effects from such oils, including the lowering of serum cholesterol and blood pressure, reducing the risk of diabetic necropathy and cardio-reperfusion injury $[8,16,17]$. Chiou et al. [18] reported that during frying, foods absorb oil, the composition of which is similar to that remaining in the frying pan. Therefore, it is vital to investigate the quality of the oils during and after the frying process. 
A number of studies have provided information on the quality deterioration of frying oils, but only a few studies focused on the stability of bioactive compounds during deep-frying [19-21]. The information regarding the antioxidant degradation kinetic and the changes of these bioactive compounds during deep-frying is relatively scarce. Hence, this study was conducted to evaluate the radical scavenging capacity and prominent bioactive compounds ( $\alpha$-tocopherol, $\gamma$-tocotrienol and $\gamma$-oryzanol) in PO and RBO, as well as to examine their degradation kinetic during the deep-frying of French fries.

\section{Experimental Section}

\subsection{Materials}

Yellow palm olein oil (Buruh Brand, Shah Alam, Malaysia), rice bran oil (Green Love Brand, Amorchai Co., Ltd., Bangkok, Thailand) and French fries (KG Brand, Shah Alam, Malaysia) were purchased from a local supermarket in Serdang, Selangor, Malaysia. Alpha-tocopherol, $\gamma$-tocotrienol and 2,2-diphenyl-1-picrylhydrazyl (DPPH) were purchased from Sigma-Aldrich, Steinheim, Germany. The gamma-oryzanol standard was purchased from Wako Pure Chemical Industries Ltd., Beijing, China. Hexane, isopropyl alcohol, acetonitrile, butanol, acetic acid and isooctane were purchased from Fisher Scientific, U.K. All other reagents and chemicals used were of analytical or HPLC grade.

\subsection{Deep-Frying Model}

A deep-frying model as described by Schroeder et al. [22] was adopted with slight modification. Frying oil with an initial volume of $3 \mathrm{~L}$ was heated to $180 \pm 5^{\circ} \mathrm{C}$ in a kitchen fryer (Philux, Model DF30AIT, Libertronic Sdn Bhd, Seri Kembangan Selangor). French fries (100 g) were then fried in the oil for $2 \mathrm{~min}$, denoted as first batch deep-frying. The fried French fries were then removed, and the deep-frying was repeated with a new batch of French fries without any time lag. This procedure was repeated until the 60th frying cycle. An aliquot of the oil $(30 \mathrm{~mL})$ was sampled after each tenth batch of French fries deep-frying to be analyzed and compared with that of fresh oil.

\subsection{Determination of Free Radical Scavenging Capacity}

The antioxidant capacity of fresh PO and RBO and after deep-frying (20th, 40th and 60th frying cycles) were determined using the 2,2-diphenyl-1-picryl hydrazyl (DPPH) radical scavenging assay as described by Lee et al. [23]. Briefly, 0.7 grams of oil samples were dissolved in $10 \mathrm{~mL}$ isooctane as a stock solution. Dissolved oil $(0.25 \mathrm{~mL})$ was then added to $1.75 \mathrm{~mL}$ DPPH solution $\left(25 \mathrm{mg} \cdot \mathrm{L}^{-1}\right.$ prepared in isooctane). The mixture was then vortexed and incubated at ambient temperature for $30 \mathrm{~min}$. The absorbance was measured at $515 \mathrm{~nm}$ using a UV-Vis spectrophotometer (EL800, Biotek, Winooski, VT, USA). The radical scavenging activity was calculated using the following formula:

$$
\text { Radical scavenging activity }(\%)=\frac{(\text { Abs Control }- \text { Abs Sample })}{\text { Abs Control }} \times 100 \%
$$


where Abs control is the absorbance of the control reaction (containing all reagents except the test compound) and Abs sample is the absorbance of the test sample. Synthetic antioxidant butylated hydroxyanisole (BHA) and $\alpha$-tocopherol were used as positive controls.

\subsection{Determination of $\alpha$-Tocopherol and $\gamma$-Tocotrienol}

Alpha-tocopherol and $\gamma$-tocotrienol were determined according to the American Oil Chemists' Society (AOCS) Ce 8-89 [24] method with slight modifications. Oil was dissolved with $10 \mathrm{~mL}$ of hexane:isopropyl alcohol (ratio 98:2) and filtered through a $0.45 \mu \mathrm{m}$ (Whatman) nylon membrane filter prior to injecting into a HPLC. An aliquot of sample $(20 \mu \mathrm{L})$ was analyzed using a normal phase HPLC system equipped with chromatography software (Millennium LC-6A, Shimadzu, Kyoto, Japan), a UV detector (SPD-6A, Shidmazu, Kyoto, Japan) and pumps. The separation was performed with an ACE 5 Silica column $(250 \times 4.6 \mathrm{~mm}, 5 \mu \mathrm{m})$ operated at ambient temperature. The mobile phase consisted of hexane:isopropyl alcohol at a ratio of 98:2(v/v) with a flow rate of $1 \mathrm{~mL} \cdot \mathrm{min}^{-1}$, and the detector was set at $295 \mathrm{~nm}$. The identification of unknown tocopherol and tocotrienol isomers was based upon matching their retention time with those of pure standards, while the concentrations calculated using the peak area based on standard calibration curves.

\subsection{Determination of $\gamma$-Oryzanol in Rice Bran Oil}

The content of $\gamma$-oryzanol was determined according to the method of $\mathrm{Xu}$ and Godber [25]. An aliquot $(0.1 \mathrm{~g})$ of oil was dissolved in $10 \mathrm{~mL}$ hexane:isopropyl alcohol at a ratio of 98:2 (v/v). The dissolved oil sample was then filtered through a $0.45 \mu \mathrm{M}$ (Whatman) nylon membrane filter prior to injecting into a HPLC. The filtered sample $(20 \mu \mathrm{L})$ was analyzed using an HPLC system (Millennium LC-6A, Shimadzu, Tokyo, Japan) chromatography manager, a UV detector (SPD-6A, Shidmazu, Tokyo, Japan) and pumps. The separation was carried using a Waters reverse-phase (RP) $\mu$ Bondapak C18 column $(250 \times 4.6 \mathrm{~mm}, 5 \mu \mathrm{M}$; Waters Corp., Milford, MA, USA $)$ at ambient temperature. The mobile phase consisted of acetonitrile/butanol/acetic acid/water at a ratio of 94:3:2:1 (v/v/v/v). The flow rate was kept at $1 \mathrm{~mL} \cdot \mathrm{min}^{-1}$ with the isocratic mode, and the detector was set at $330 \mathrm{~nm}$. Identification of the $\gamma$-oryzanol component in the RBO was done by comparing its retention with that of pure standard, and quantification was done based on the standard calibration curve.

\subsection{Determination of Degradation Kinetics Using the Order of Reaction Equation}

According to Taoukis et al. [26], the degradation kinetics of many compounds in foods at constant temperature follows the first-order kinetics model, which can be expressed as follows:

$$
-\mathrm{dC} / \mathrm{dt}=\mathrm{kC}
$$

Integration of Equation 1 gives:

$$
\begin{gathered}
\operatorname{lnC}=\operatorname{lnCo}-\mathrm{kt} \\
\ln \mathrm{C} / \mathrm{Co}=-\mathrm{kt}
\end{gathered}
$$


where $\mathrm{C}$ is the concentration of the compound, $\mathrm{Co}$ is the initial concentration of the compound, $\mathrm{t}$ is time and $\mathrm{k}$ is the reaction rate constant. By plotting the logarithm of the concentration of $\alpha$-tocopherol and $\gamma$-tocotrienol, respectively, over the initial concentration against a number of frying cycles during the degradation process, the degradation rate constant for each compound was calculated from the slope of the simple linear regression line.

\subsection{Statistical Analysis}

All frying experiments and analyses were conducted in triplicate. Data was analyzed statistically through one-way ANOVA by Duncan's multiple range tests using the commercially available software, the SPSS 16 software program (SPSS Inc., Chicago, IL, USA). A $p$-value of less than 0.05 $(p<0.05)$ was considered to denote statistical significance.

\section{Results and Discussion}

\subsection{Antioxidant Capacity of the Oils}

In this study, free radical scavenging activity utilizing the DPPH radical was done in determining the antioxidant capacity of the oils, due to its simplicity and wide use in antioxidant research. The free radical scavenging capacity of PO and RBO after the 20th, 40th and 60th frying cycle is shown in Figure 1. Results showed that there is no significant difference in the antioxidant capacity between the fresh and the 20th frying cycle PO. However, a significant $(p<0.05)$ decrease in the activity was observed for the oil from the 40th and 60th frying cycle. On the other hand, there was no significant difference in the antioxidant activity exhibited by all the batches of RBO. This indicates that RBO is more stable compared to that of PO upon deep-frying. It was also noted that RBO derived from the 40th and 60th frying cycle exhibited significantly $(p<0.05)$ higher radical scavenging activity than that of PO.

The radical scavenging assay is one of the common methods that can be used to determine the antioxidant capacity of vegetable oils [27]. The results of the study showed that both PO and RBO exhibited good antioxidant capacity. However, the antioxidant capacity of PO decreased with deep-frying. This is probably attributed to the degradation of bioactive compounds as a result of the exposure to high temperature used during deep-frying. Results presented here are in line with that of a previous study conducted by Gomez-Alonso et al. [28], where the reduction of antioxidant activity of olive oil correlated well with the number of deep-frying cycles. However, similar the destruction of some of these bioactive compounds may not be occurring in RBO, as the antioxidant activity in the oil was preserved throughout the frying process.

\subsection{Degradation of $\alpha$-Tocopherol and $\gamma$-Tocotrienol during Deep-Frying}

The degradation of $\alpha$-tocopherol in PO and RBO during deep-frying is shown in Figure 2. As expected, results showed that the concentration of $\alpha$-tocopherol in PO decreased significantly with deep-frying. Similar to that of its antioxidant activity, $\alpha$-tocopherol content decreased from $1.29 \mathrm{mg} \cdot \mathrm{g}^{-1}$ to $0.59 \mathrm{mg} \cdot \mathrm{g}^{-1}$ after the 60 th frying cycle, depicting a loss of $54 \%$. Surprisingly, $\alpha$-tocopherol in RBO was found to be more stable and remained almost unaffected upon deep-frying up to the 60th frying 
cycle. The contents of $\alpha$-tocopherol in fresh RBO and the 60th frying cycle were $0.75 \mathrm{mg} \cdot \mathrm{g}^{-1}$ and $0.73 \mathrm{mg} \cdot \mathrm{g}^{-1}$, respectively, a loss of only $2 \%$. This is reflected in the antioxidant activity exhibited by the oil. The degradation of $\alpha$-tocopherol in PO is in agreement with that of previous literature $[14,15]$. Battino et al. [14] found that $\alpha$-tocopherol in olive oil was degraded up to $28 \%$ from the initial value after frying for $60 \mathrm{~min}$.

Figure 1. The percentage of radical-scavenging activity in palm oil and rice bran oil over the 60 th batch of deep-frying at a concentration of $70 \mathrm{mg} / \mathrm{mL}$. The values given are means \pm standard deviation of a triplicate analysis. The values marked with the same letters are not significantly different at $p<0.05$ analyzed using the Duncan multiple range test. PO, palm oil; RBO, rice bran oil.

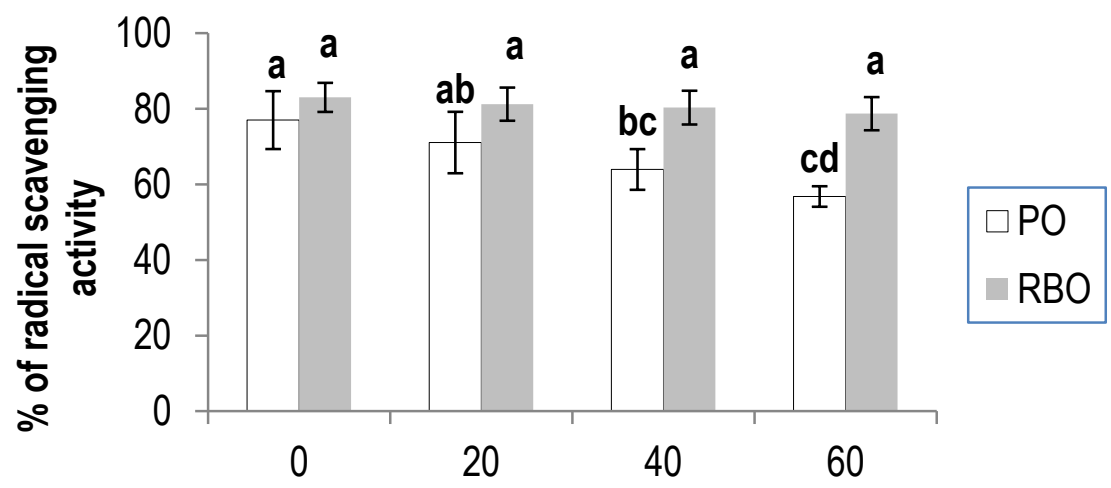

No. of frying cycles

Figure 2. The degradation of $\alpha$-tocopherol in palm oil and rice bran oil during deep-frying. The values given are means \pm standard deviation of triplicate analysis. The values marked with the same letters are not significantly different at $p<0.05$ analyzed using the Duncan multiple range test. A,B, the values with different capital letters indicate a significant difference between the type of oil at $p<0.05$. a,b, the values with different small letters indicate a significant difference between frying cycle numbers at $p<0.05$.

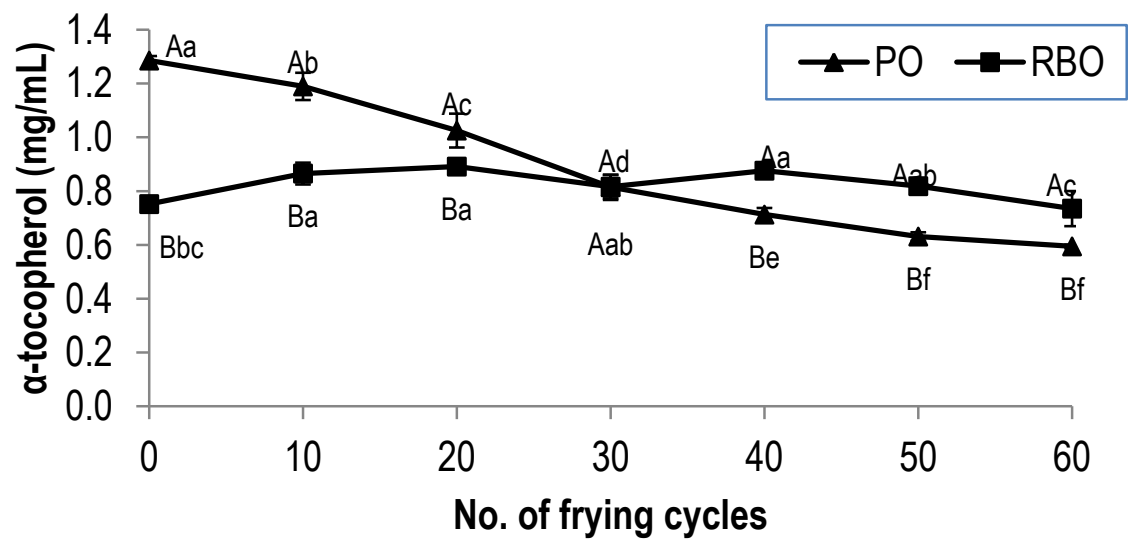

Figure 3 showed the degradation of $\gamma$-tocotrienol in deep-fried PO and RBO. There was a similar trend on the loss of $\gamma$-tocotrienol in both oils during the deep-frying of French fries, where it decreased significantly with frying. However, interestingly, $\gamma$-tocotrienol's content in RBO was always higher 
than that of PO in all batches of oils. The fresh PO and the 60th frying cycle PO consisted of $0.79 \mathrm{mg} \cdot \mathrm{g}^{-1}$ and $0.28 \mathrm{mg} \cdot \mathrm{g}^{-1}$ tocotrienol, respectively depicting a loss of $65 \%$. Whereas, the content of $\gamma$-tocotrienol in fresh RBO and the 60th frying cycles of RBO were $1.34 \mathrm{mg} \cdot \mathrm{g}^{-1}$ and $0.77 \mathrm{mg} \cdot \mathrm{g}^{-1}$, respectively, showing a lower loss of $42 \%$.

Figure 3. The degradation of $\gamma$-tocotrienol in palm oil and rice bran oil during deep-frying. The values given are means \pm standard deviation of a triplicate analysis. The values marked with the same letters are not significantly different at $p<0.05$ analyzed using the Duncan multiple range test. A,B, the values with different capital letters indicate a significant difference between the type of oil at $p<0.05$. a,b, the values with different small letters indicate a significant difference between frying cycle numbers at $p<0.05$.

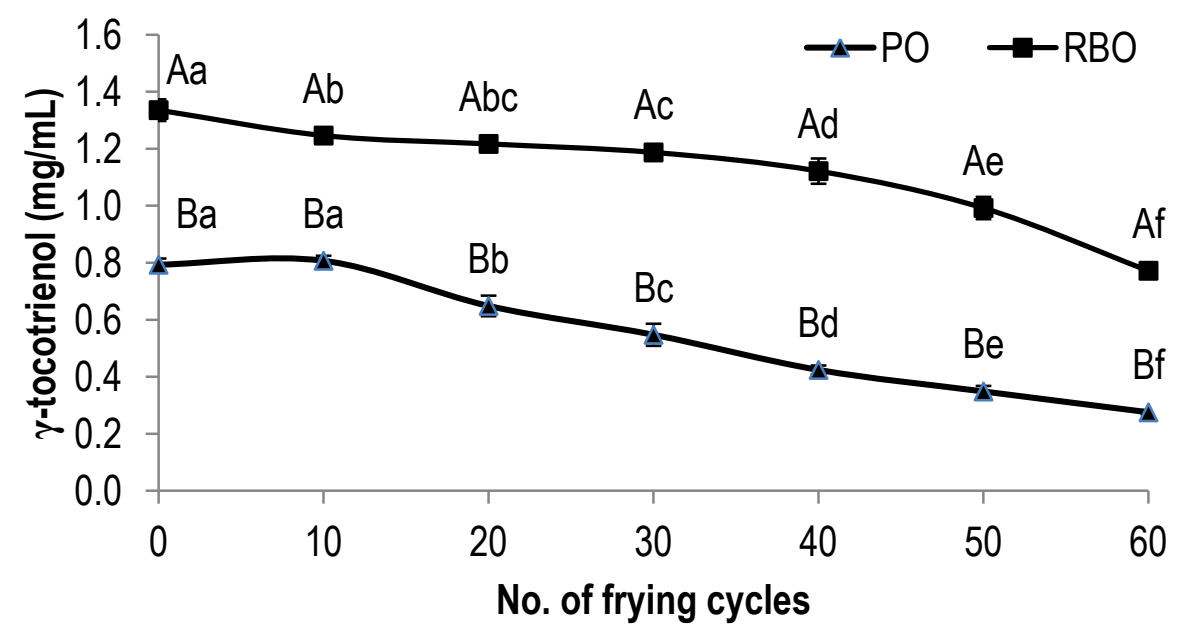

During the deep-frying of fries, the decrease in the concentration of tocopherols and tocotrienols in PO might have occurred due to their protective role against oxidation and degradation upon exposure to high temperature. A similar observation has been reported by Hoffmann [29], where at a high temperature, tocopherols and tocotrienols tend to protect the oil by oxidizing themselves to quinones and dimers. The present result also indicated that $\alpha$-tocopherol and $\gamma$-tocotrienol in PO degraded at a faster rate than that of RBO. Generally, PO consisted of an equal amount of saturated fatty acid to unsaturated fatty acid, whereas RBO contained a higher amount of unsaturated fatty acid, usually up to $74 \%$. A higher proportion of unsaturated fatty acids in RBO, relative to PO, could be correlated to the greater number of double bonds in the former oil [21,22]. Therefore, it can be suggested that during the deep-frying of RBO, these unsaturation might have competed with tocopherols and tocotrienols as substrates for oxidation, resulting in a slower degradation of these antioxidants. In addition, a higher degree of unsaturation, as a basis of more competitiveness of the fatty acids towards oxidation, may explain the high degradation rate of $\alpha$-tocopherol and $\gamma$-tocotrienol in PO as compared with that of RBO [30]. This is in agreement with that reported by Hoffmann [29], who showed that during the propagation phase of oxidation, the fatty acid peroxy free radicals may react preferentially with the phenolic hydrogen molecule of tocopherol. Seppanen et al. [31] reported that the antioxidant activities of tocopherols and tocotrienols in oils are mainly due to their abilities to donate phenolic hydrogen to reactive lipid free radicals, thus retarding the normal steps of autocatalytic lipid peroxidation. 
Tocopherols can effectively scavenge peroxy radicals and yield relatively stable products, which interrupt the propagation stage of the oxidative chain reaction, thereby preventing the destruction of lipid molecules [32,33].

It is interesting to note that the concentration of $\alpha$-tocopherol in RBO was maintained during the deep-frying of the fries exhibiting only a low percent loss (Figure 2). In contrast, the content of $\gamma$-tocotrienol in RBO decreased considerably with increased frying. In RBO, $\gamma$-tocotrienol is expected to possess higher antioxidant activity than that of $\alpha$-tocopherol. This was also applied to PO, which exerts the same trend [22]. Therefore, it can be suggested that $\gamma$-tocotrienol was firstly oxidized in order to protect other weak antioxidants. This is in agreement with that reported by Rossi et al. [30], where among vitamin E homologs, $\gamma$-tocotrienol was found to be the least stable and easily oxidized during deep-frying, which might be attributed mainly to its protective role and self-degradation in preserving other homologues, such as tocopherols. Apart from that, the initial content of $\gamma$-tocotrienol in fresh RBO was significantly higher than that in PO, although the concentration of both decreased with an increase in the number of frying cycles. Therefore, with a higher concentration, the protective effect of $\gamma$-tocotrienol in preserving $\alpha$-tocopherol was evident.

\subsection{Degradation of $\gamma$-Oryzanol in Rice Bran Oil during Deep-Frying}

Figure 4 showed the degradation of $\gamma$-oryzanol in RBO over the 60th frying cycles. The concentration of $\gamma$-oryzanol in RBO was seen to decrease significantly $(p<0.05)$ in accordance to the number of frying batches. Fresh oil, containing $3.0 \mathrm{mg} \cdot \mathrm{g}{ }^{-1} \gamma$-oryzanol, was reduced to $2.8 \mathrm{mg} \cdot \mathrm{g}^{-1}$ at the 20th cycles, $2.6 \mathrm{mg} \cdot \mathrm{g}^{-1}$ after 40 cycles and $2.4 \mathrm{mg} \cdot \mathrm{g}^{-1}$ after 60 batches. The remaining $\gamma$-oryzanol content in RBO after the 60th frying cycles was estimated to be $80 \%$ of the original amount in the fresh oil.

RBO naturally contains $\alpha$-tocopherol, $\gamma$-tocotrienol and $\gamma$-oryzanol, making it a suitable model to study the synergistic interaction between these bioactive compounds in protecting lipids from oxidation. The ability of a compound to inhibit the oxidation process is via several mechanisms. Gamma-oryzanol is one of the most potent antioxidant compounds reported. It is interesting to note that besides acting as an antioxidant to prevent oil from oxidation, $\gamma$-oryzanol is also associated with decreasing cholesterol in plasma, platelet aggregation and cholesterol absorption [34-36]. In addition, $\gamma$-oryzanol has also been used to treat nerve imbalance and disorders of menopause in women [37].

The results in this study indicated that $\gamma$-oryzanol in RBO was degraded with the number of frying cycles, and its degradation is parallel to that of $\gamma$-tocotrienol. However, the $\alpha$-tocopherol content in RBO did not decrease significantly $(p<0.05)$ until the 60th frying cycles (Figure 2$)$. Thus, it can be assumed that, in RBO, $\gamma$-oryzanol and $\gamma$-tocotrienol synergistically protect $\alpha$-tocopherol from degradation during deep-frying. The presence of $\gamma$-oryzanol in RBO may confer a protective effect for $\alpha$-tocopherol, thus slowing down its degradation compared to that in PO. This observation was in agreement with that of Kochhar [38], who reported that $\gamma$-oryzanol has substantial synergistic effects with tocopherols. Similarly, the higher amount of $\gamma$-oryzanol, due to the addition of RBO to soybean oil, was related to the synergistic antioxidant effects in preserving tocopherol degradation during the frying of dough [39]. 
Figure 4. The degradation of $\gamma$-oryzanol in RBO during deep-frying. The values given are means \pm standard deviation of a triplicate analysis. The values marked with the same letters are not significantly different at $p<0.05$ analyzed using the Duncan multiple range test.

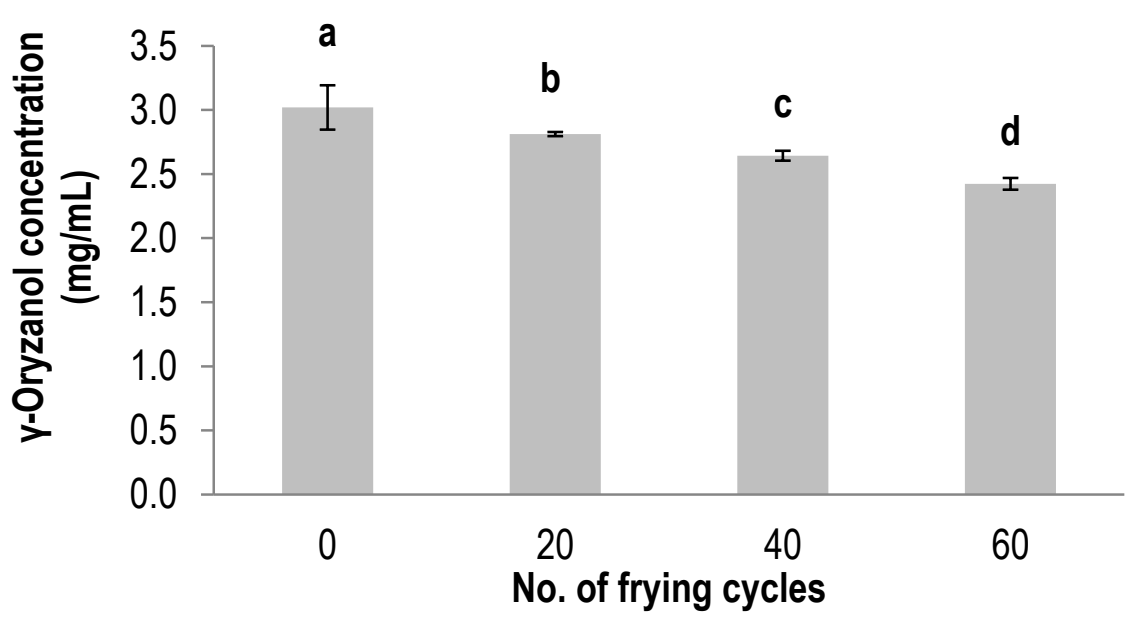

RBO usually contains oryzanol up to $1.5 \%$ and is well known for its good stability as a frying oil [6]. Even though RBO contained a higher percent of $\gamma$-oryzanol in comparison to that of $\alpha$-tocopherol and $\gamma$-tocotrienol, the main reaction that caused the degradation of $\gamma$-oryzanol during deep-frying is due to the oxidation process [40]. In the present study, the antioxidant capacity of PO as measured by DPPH decreased significantly $(p<0.05)$ after deep-frying, similar to its $\alpha$-tocopherol and $\gamma$-tocotrienol degradation. In contrast, the antioxidant capacity of RBO did not decrease significantly over 60 frying cycles. The slow degradation of $\gamma$-tocotrienol and the retention of $\alpha$-tocopherol in RBO was probably attributed to the preservation of its antioxidant activity. This is in agreement with the findings of Nanua et al. [41], who reported that $\gamma$-oryzanol can improve the stability of RBO and acts as a natural antioxidant to improve the stability of several fried foods. Hence, it is suggested that blending PO with RBO might be helpful to preserve the tocol content during deep-frying.

\subsection{Degradation Kinetic of $\alpha$-Tocopherol and $\gamma$-Tocotrienol during Deep-Frying}

The first order kinetic plots of the degradation of $\alpha$-tocopherol and $\gamma$-tocotrienol in PO and RBO during deep-frying of French fries are presented in Figures 5 and 6, respectively. The kinetic constant of degradation $(k)$ of $\alpha$-tocopherol and $\gamma$-tocotrienol and the determination of the coefficient $\left(R^{2}\right)$ for $\mathrm{PO}$ and RBO during deep-frying is shown in Table 1.

The results showed that the first-order kinetics model can be applied to approximately describe the degradation reaction of $\alpha$-tocopherol in PO, but not in RBO. It is reported that the degradation kinetics of many compounds in foods at constant temperature will follow the first-order kinetics model [26]. This is in good agreement with the findings of Sabliov et al. [42], where heat during frying caused the degradation of free $\alpha$-tocopherol followed first order kinetics and holding oils at $180 \pm 5{ }^{\circ} \mathrm{C}$ showed the greatest degradation rate. 
Figure 5. First order kinetic plots of the degradation of $\alpha$-tocopherol in palm oil and rice bran oil during deep-frying.

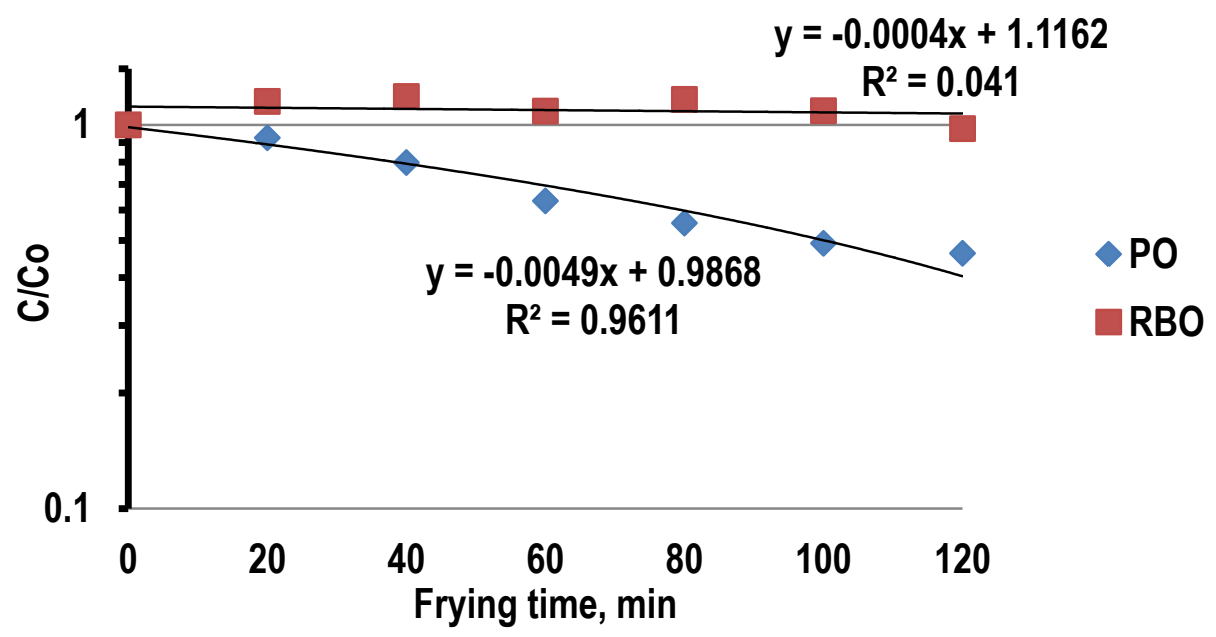

Figure 6. First order kinetic plots of the degradation of $\gamma$-tocotrienol in palm oil and rice bran oil during deep-frying.

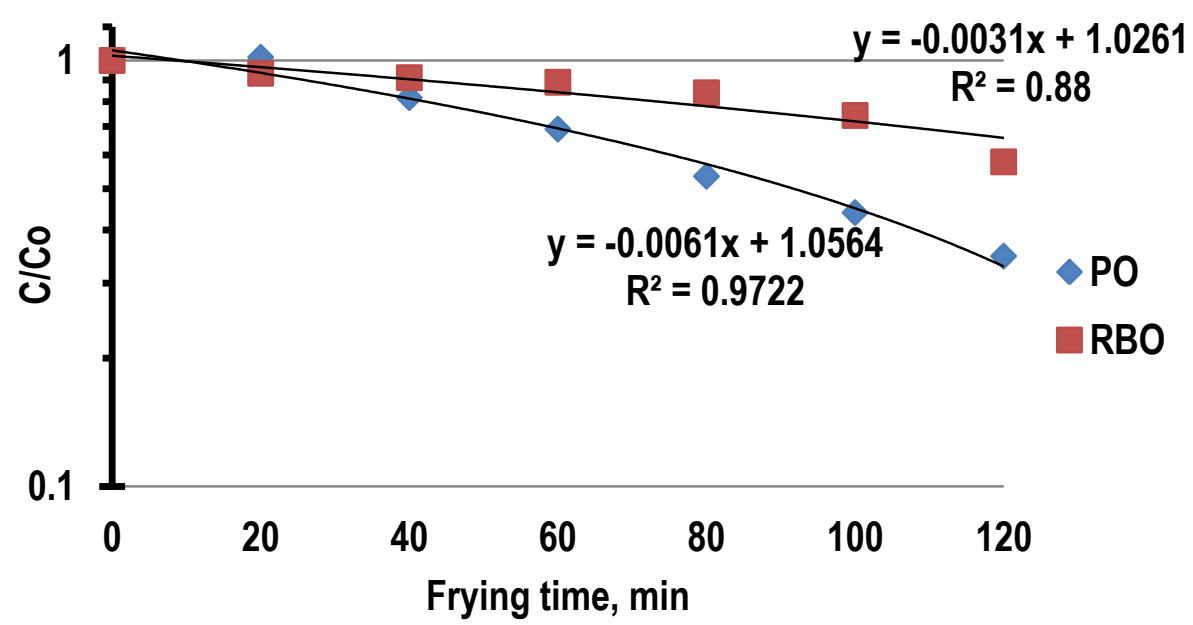

Table 1. Kinetic constant $(k)$ of degradation of $\alpha$-tocopherol and $\gamma$-tocotrienol and the coefficient of determination $\left(r^{2}\right)$ of palm oil and rice bran oil during deep-frying.

\begin{tabular}{ccc}
\hline Variable & $\boldsymbol{k}\left(\mathbf{m i n}^{-\mathbf{1}}\right)$ & Coefficient of Determination, $\left.\boldsymbol{r}^{\mathbf{2}}\right)$ \\
\hline$\alpha$-tocopherol in palm oil & 0.004 & 0.961 \\
$\alpha$-tocopherol in rice bran oil & 0.000 & 0.041 \\
$\gamma$-tocotrienol in palm oil & 0.006 & 0.972 \\
$\gamma$-tocotrienol in rice bran oil & 0.003 & 0.880 \\
\hline
\end{tabular}

In the present study, the reaction rate constant for the degradation of both $\alpha$-tocopherol and $\gamma$-tocotrienol upon deep-frying can be obtained using the order of reaction equation. The reaction rate 
constants of the degradation of $\alpha$-tocopherol in PO and RBO were found to be $0.009 \mathrm{~min}^{-1}$ and $0 \mathrm{~min}^{-1}$, respectively and their correlation coefficients $\left(r^{2}\right)$ were found to be 0.961 and 0.041 , respectively.

Similarly, $\gamma$-tocotrienol in PO and RBO obeyed the first-order kinetics with the reaction rate constants of degradation being $0.0061 \mathrm{~min}^{-1}$ and $0.0031 \mathrm{~min}^{-1}$, respectively, and their correlation coefficients were found to be 0.972 and 0.88 , respectively. The results are in line with that of previous studies that showed that tocopherols and tocotrienols decrease as a function of time and temperature, following first-order kinetics [43]. Results from the present study showed that $\gamma$-tocotrienol in PO $\left(k=0.0061 \mathrm{~min}^{-1}\right)$ degraded faster than that of $\alpha$-tocopherol in PO $\left(k=0.0049 \mathrm{~min}^{-1}\right)$. In addition, it was reported that the antioxidant property of $\gamma$-tocotrienol is better than that of $\alpha$-tocopherol [22]. Similarly, the antioxidant capacity of $\gamma$-tocotrienol under heating conditions in stripped oils is better than that of $\alpha$-tocopherol [44]. Therefore, it could be suggested that the faster degradation of $\gamma$-tocotrienol could be due to its greater antioxidant activity.

\section{Conclusions}

Based on the present study, it is concluded that PO and RBO are good sources of natural antioxidants that include tocols and oryzanols. These compounds exhibited degradation at a faster rate in PO compared to that of RBO, with the degradation kinetics obeying the first-order kinetics. It was also noted that $\alpha$-tocopherol does not degrade to a significant extent in RBO by virtue of the presence of $\gamma$-oryzanol, indicating the synergy between the bioactive compounds in maintaining the nutritional value of the oil.

\section{Acknowledgments}

The authors would like to acknowledge The Ministry of Science, Technology and Innovation (MOSTI) of Malaysia for financing the project and the Faculty of Food Science and Technology, Universiti Putra Malaysia, for providing the laboratory facilities.

\section{Author Contributions}

This project was conducted by Evelyn Koh Wee Fang with the guidance from Mohd Sabri Mohd Pak Dek who also technically assisted in statistical analysis. Meanwhile, the kinetic study was assisted by Mohd Asraf Mohd Zainudin. Azizah Abd Hamid, senior lecturer in Functional Food and Food Chemistry subjects was the main supervisor for this project and co-supervised by Chin Ping Tan who is expert in Fats and Oil field. All authors contributed to and have approved the final manuscript.

\section{Conflicts of Interest}

The authors declare no conflict of interest.

\section{References}

1. Szydłowska-Czerniak, A.; Trokowski, K.; Karlovits, G.; Szłyk, E. Effect of refining processes on antioxidant capacity, total contents of phenolics and carotenoids in palm oils. Food Chem. 2011, $129,1187-1192$. 
2. Lam, M.K.; Tan; K.T.; Lee, K.T.; Mohamed, A.R. Malaysian palm oil: Surviving the food versus fuel dispute for a sustainable future. Renew. Sustain. Energy Rev. 2009, 13, 1456-1464.

3. Sugano, M.; Tsuji, E. Rice bran oil and cholesterol metabolism. J. Nutr. 1997, 127, S521-S524.

4. Xu, Z.; Godber, J.S. Purification and identification of components of gamma-oryzanol in rice bran oil. J. Agric. Food Chem. 1999, 47, 2724-2728.

5. Iqbal, S.; Bhanger, M.I.; Anwar, F. Antioxidant properties and components of some commercially available varieties of rice bran in Pakistan. Food Chem. 2005, 93, 265-272.

6. Norton, R.A. Quantitation of steryl ferulate and $p$-coumarate esters from corn and rice. Lipids 1995, 30, 269-275.

7. Lerma-García, M.J.; Herrero-Martínez, J.M.; Simó-Alfonso, E.F.; Mendonça, C.R.B.; Ramis-Ramos, G. Composition, industrial processing and applications of rice bran $\gamma$-oryzanol. Food Chem. 2009, 115, 389-404.

8. Siddiqui, S.; Rashid Khan, M.; Siddiqui, W.A. Comparative hypoglycemic and nephroprotective effects of tocotrienol rich fraction (TRF) from palm oil and rice bran oil against hyperglycemia induced nephropathy in type 1 diabetic rats. Chem. Biol. Interact. 2010, 188, 651-658.

9. Müller, L.; Theile, K.; Böhm, V. In vitro antioxidant activity of tocopherols and tocotrienols and comparison of vitamin E concentration and lipophilic antioxidant capacity in human plasma. Mol. Nutr. Food Res. 2010, 54, 731-742.

10. Chelchowska, M.; Ambroszkiewicz, J.; Gajewska, J.; Laskowska-Klita, T.; Leibschang, J. The effect of tobacco smoking during pregnancy on plasma oxidant and antioxidant status in mother and newborn. Eur. J. Obstet. Gynecol. Reprod. Biol. 2011, 155, 132-136.

11. Sakurai, H.; Yoshihashi, T.; Nguyen, H.T.T.; Pokorn, J. A new generation of frying oils. Czech. J. Food Sci. 2003, 21, 145-151.

12. Yamsaengsung, R.; Moreira, R.G. Modeling the transport phenomena and structural changes during deep fat frying. Part II: Model solution \& validation. J. Food Eng. 2002, 53, 11-25.

13. Choe, E.; Min, D.B. Chemistry of deep-fat frying oils. J. Food Sci. 2007, 72, R77-R86.

14. Battino, M.; Quiles, J.L.; Huertas, J.R.; Ramirez-Tortosa, M.C.; Cassinello, M.; Mañas, M.; Lopez-Frias, M.; Mataix, J. Feeding fried oil changes antioxidant and fatty acid pattern of rat and affects rat liver mitochondrial respiratory chain components. J. Bioenerg. Biomembr. 2002, 34, $127-134$.

15. Quiles, J.L.; Huertas, J.R.; Battino, M.; Ramìrez-Tortosa, M.C.; Cassinello, M.; Mataix, J.; Lopez-Frias, M.; Mañas, M. The intake of fried virgin olive or sunflower oils differentially induces oxidative stress in rat liver microsomes. Br. J. Nutr. 2002, 88, 57-65.

16. Raederstorff, D.E.; Elste, V.; Aebischer, C.; Weber, P. Effect of either $\gamma$-tocotrienol or a tocotrienol mixture on plasma lipid profile in hamster. Ann. Nutr. Metab. 2002, 46, 17-23.

17. Esterhuyse, J.S.; van Rooyen, J.; Strijdom, H.; Bester, D.; du Toit, E.F. Proposed mechanisms for red palm oil induced cardioprotection in a model of hyperlipidaemia in the rat. Prostaglandins Leukot. Essent. Fatty Acids 2006, 75, 375-384.

18. Chiou, A.; Kalogeropoulos, N.; Salta, F.N.; Efstathiou, P.; Andrikopoulos, N.K. Pan-frying of French fries in three different edible oils enriched with olive leaf extract: Oxidative stability and fate of microconstituents. Food Sci. Technol. 2009, 42 1090-1097. 
19. Juárez, M.D.; Osawa, C.C.; Acuña, M.E.; Sammán, N.; Gonçalves, L.A.G. Degradation in soybean oil, sunflower oil and partially hydrogenated fats after food frying, monitored by conventional and unconventional methods. Food Control 2011, 22, 1920-1927.

20. Bou, R.; Navas, J.A.; Tres, A.; Codony, R.; Guardiola, F. Quality assessment of frying fats and fried snacks during continuous deep-fat frying at different large-scale producers. Food Control 2012, 27, 254-267.

21. Debnath, S.; Rastogi, N.K.; Gopala Krishna, A.G.; Lokesh, B.R. Effect of frying cycles on physical, chemical and heat transfer quality of rice bran oil during deep-fat frying of poori: An Indian traditional fried food. Food Bioprod. Process. 2012, 90, 249-256.

22. Schroeder, M.T.; Becker, E.M.; Skibsted, L.H. Molecular mechanism of antioxidant synergism of tocotrienols and carotenoids in palm oil. J. Agric. Food Chem. 2006, 54, 3445-3453.

23. Lee, Y.L.; Huang, G.W.; Liang, Z.C.; Mau, J.L. Antioxidant properties of three extracts from Pleurotus citrinopileatus. LWT-Food Sci. Technol. 2007, 40, 823-833.

24. AOCS Ce 8-89. Determination of tocopherols and tocotrienols in vegetable oils and fats by HPLC. In Official Methods and Recommended Practices of the American Oil Chemists' Society, 5th ed.; American Oil Chemists' Society: Champaign, IL, USA, 1998.

25. Xu, Z.; Godber, J.S. Antioxidant activities of major components of $\gamma$-oryzanol from rice bran using a linoleic acid model. J. Am. Oil Chem. Soc. 2001, 78, 645-649.

26. Taoukis, P.S.; Labuza, T.P.; Saguy, I.S. Kinetics of food deterioration and shelf-life prediction. In Handbook of Food Engineering Practice; Valentas, K.J., Rotstein, E., Singh, R.P., Eds.; CRC Press: Boca Raton, FL, USA, 1997; pp. 261-402.

27. Ramadan, M.F.; Amer, M.M.A.; Sulieman, A.E.M. Correlation between physicochemical analysis and radical scavenging activity of vegetable oil blends as affected by frying of French fries. Eur. J. Lipid Sci. Technol. 2006, 108, 670-678.

28. Gomez-Alonso, S.; Fregapane, G.; Salvador, M. Changes in phenolic composition and antioxidant activity of virgin olive oil during frying. J. Agric. Food Chem. 2003, 51, 667-672.

29. Hoffmann, G. The chemistry of edible fats. In The Chemistry and Technology of Edible Oils and Fats and Their High Fat Products; Hoffmann, G., Ed.; Academic Press: London, UK, 1998; pp. 1-28.

30. Rossi, M.; Alamprese, C.; Ratti, S. Tocopherols and tocotrienols as free radical-scavengers in refined vegetable oils and their stability during deep-fat frying. Food Chem. 2007, 102, 812-817.

31. Seppanen C.M.; Song Q.H.; Csallany A.S. The antioxidant functions of tocopherol and tocotrienol homologues in oils, fats, and food systems. J. Am. Oil Chem. Soc. 2010, 87, 469-481.

32. Fang, X.; Wada, S. Enhancing the antioxidant effect of $\alpha$-tocopherol with rosemary in inhibiting catalyzed oxidation caused by $\mathrm{Fe}^{2+}$ and hemoprotein. Food Res. Int. 1993, 26, 405-411.

33. Michael, H.; Kourimska, G.; Kourimska, L. Effect of antioxidants on losses of tocopherols during deep-fat frying. Food Chem. 1995, 52, 175-177.

34. Lichenstein, A.H.; Ausman, L.M.; Carrasco, W.; Gualtieri, L.J.; Jenner, J.L.; Ordovas, J.M.; Nicolosi, R.J.; Goldin, B.R.; Schaefer, E.J. Rice bran oil consumption and plasma lipid levels in moderately hypercholesterolemic humans. Arterioscler. Thromb. 1994, 14, 549-556.

35. Seetharamaiah, G.S.; Krishnakantha, T.P.; Chandrasekhara, N. Influence of oryzanol on platelet aggregation in rats. J. Nutr. Sci. Vitaminol. 1990, 36, 291-297. 
36. Rong, N.; Ausman, L.M.; Nicolosi, R.J. Oryzanol decreases cholesterol absorption and aortic fatty streaks in hamsters. Lipids 1997, 32, 303-309.

37. Nakayama, S.; Manabe, A.; Suzuki, J.; Sakamoto, K.; Inagake, T. Comparative effects of two forms of $\gamma$-oryzanol in different sterol compositions on hyperlipidemia induced by cholesterol. Jpn. J. Pharmacol. 1987, 44, 135-143.

38. Kochhar, S.P. Stabilisation of frying oils with natural antioxidative components. Eur. J. Lipid Sci. Technol. 2000, 102, 552-559.

39. Chotimarkorn, C.; Silalai, N. Addition of RBO to soybean oil during frying increases the oxidative stability of the fried dough from rice flour during storage. Food Res. Int. 2008, 41, 308-317.

40. Khuwijitjaru, P.; Taengtieng, N.; Changprasit, S. Degradation of gamma-oryzanol in rice bran oil during heating: An analysis using derivative UV-spectrophotometry. Silpakorn Univ. Int. J. 2004, 4, 154-165.

41. Nanua, J.N.; McGregor, J.U.; Godber, J.S. Influence of high-oryzanol RBO on the oxidative stability of whole milk powder. J. Dairy Sci. 2000, 83, 2426-2431.

42. Sabliov, C.M.; Fronczek, C.; Astete, C.E.; Khachaturyan, M.; Khachatryan, L.; Leonardi, C.; Brazzoli, I. Effects of temperature and $u v$ light on degradation of a-tocopherol in free and dissolved form. J. Am. Oil Chem. Soc. 2009, 86, 895-902.

43. Alyssa, H.; Andrea, B.; Carlo, P. Kinetics of tocols degradation during the storage of einkorn (Triticum monococcum L. ssp. Monococcum) and breadwheat (Triticum aestivum L. ssp. aestivum) flours. Food Chem. 2009, 116, 821-827.

44. Feng, H.P. Preparative Techniques for Isolation of Vitamin E Homologs and Evaluation of Their Antioxidant Activities. Ph.D. Thesis, Atherns, University of Georgia, Athens, GA, USA, 1996.

(C) 2014 by the authors; licensee MDPI, Basel, Switzerland. This article is an open access article distributed under the terms and conditions of the Creative Commons Attribution license (http://creativecommons.org/licenses/by/3.0/). 